\title{
Laboratory Evaluation of Four Different Devices for Secretion Mobilization: Acapella Choice, Green and Blue Versus Water Bottle
}

\author{
Gabi Mueller PhD, Ines Bersch-Porada PT MSc, Sabrina Koch-Borner PT, \\ Anja M Raab PT MSc, Marga Jonker PT, Michael Baumberger MD, and Franz Michel MD
}

\begin{abstract}
BACKGROUND: Secretion removal is a key issue in patients with respiratory diseases, and is known to be most effective at vibration frequencies of $\sim 13 \mathrm{~Hz}$ and with the greatest amplitudes possible. The Acapella devices and the water bottle are used for secretion removal in daily clinical practice but without detailed knowledge on optimal settings. The aim of this study was to evaluate the 3 different Acapella devices and the water bottle at various settings and flows to determine the optimal devices and settings for effective secretion removal. METHODS: Three different Acapella devices were tested at flows of $6,12,20,30,40$, and $50 \mathrm{~L} / \mathrm{min}$, and at all 5 settings. The water bottle was filled with 5,10 , or $15 \mathrm{~cm}$ of water, and tested at flows of $3,6,10,12$, and $20 \mathrm{~L} / \mathrm{min}$. For all devices and combinations of settings, we measured the frequency and amplitude of the vibrations, as well as the required pressure to generate vibrations. RESULTS: Setting 4 was the best for all 3 Acapella devices, and the filling height of the water bottle should be $5 \mathrm{~cm}$. At these settings, all devices elicited vibration frequencies between 12 and $15 \mathrm{~Hz}$, which is theoretically optimal for secretion mobilization. The resistance pressures of the devices to elicit these vibrations were between 5 and $11 \mathrm{~cm} \mathrm{H}_{2} \mathrm{O}$. However, the Acapella devices elicit higher vibration amplitudes $(5-8 \mathrm{~cm}$ $\left.\mathrm{H}_{2} \mathrm{O}\right)$ than the water bottle $\left(1.8 \mathrm{~cm} \mathrm{H} \mathrm{H}_{2} \mathrm{O}\right)$ CONCLUSIONS: Setting 4 was optimal for all 3 Acapella devices. The Acapella devices may be more efficient for secretion mobilization than the water bottle, because they elicit greater amplitude of vibrations. Key words: airway clearance therapy; bronchial clearance; chest physiotherapy; evaluation of devices; mисиs; oscillating positive expiratory pressure; respiratory secretions. [Respir Care 2014;59(5):673-677. (C) 2014 Daedalus Enterprises]
\end{abstract}

\section{Introduction}

Secretion clearance is of utmost importance in conditions with excessive production of sputum (eg, cystic fibrosis) or secretion retention as a result of weak or paralyzed respiratory muscles (eg, multiple sclerosis, amyo-

Dr. Mueller is affiliated with the Clinical Trial Unit; Mss. Bersch-Porada, Koch-Borner, Raab, and Jonker are affiliated with Physiotherapy; Dr. Baumberger is affiliated with the Clinic; and Dr. Michel is affiliated with Pneumology, Swiss Paraplegic Centre, Nottwil, Switzerland.

The authors have disclosed no conflicts of interest.

Correspondence: Dr. Gabi Mueller, Clinical Trial Unit; Swiss Paraplegic Centre, CH-6207 Nottwil, Switzerland. E-mail: gabi.mueller@paraplegie.ch.

DOI: $10.4187 /$ respcare.02654 trophic lateral sclerosis, spinal cord injury). There are different devices for secretion mobilization (eg, Flutter, Acapella, water bottle, or chest vibration vests) with various requirements for their successful use. To use the devices effectively, it is very important to know specific requirements (eg, the required expiratory pressures and flows) and also the specific vibration frequencies and amplitudes of the device or setting. The applied vibration frequency is most effective for secretion mobilization when it matches the frequency of the ciliary movement, which is $\sim 13 \mathrm{~Hz} .{ }^{1}$ Furthermore, the greater the amplitude of the vibrating air, the better the effectiveness of the therapy. ${ }^{2}$ Although these parameters are critical for the effective use of a device for secretion mobilization, it is uncommon to find this information in the user manuals of the devices.

In some studies the vibration frequency and amplitude of the Flutter ${ }^{2,3}$ and the Acapella ${ }^{3-5}$ devices have been 
evaluated at different settings. However, only 2 of the 3 different Acapella devices (blue, green, and Choice) have been evaluated in the above-mentioned studies. There is no literature on the characteristics of the Acapella Choice device, and not all of the 5 settings of the "green" and the "blue" Acapella devices have been investigated. ${ }^{3}$

The water bottle is an inexpensive and readily available tool for secretion mobilization, and is often used in clinical practice. The only things needed are a $1.5 \mathrm{~L}$ plastic bottle filled with some water and a flexible tube. If it was effective, the water bottle would also be an interesting tool in developing countries with limited financial resources and limited access to devices such as the Flutter or the Acapella. However, the settings for effective secretion mobilization (ie, water quantity, flows, and tube positioning for producing frequencies of $\sim 13 \mathrm{~Hz}$ with great amplitude oscillations) are not known.

There is a general agreement in the literature that it is necessary to perform new studies evaluating oscillatory devices for secretion mobilization. ${ }^{2-4,6,7}$ Therefore, the aim of this laboratory evaluation was to determine the optimal settings for the 3 Acapella devices as well as for the water bottle, for reaching oscillating frequencies of $\sim 13 \mathrm{~Hz}$ with the greatest possible amplitudes.

\section{Methods}

\section{Acapella}

The Acapella devices (DHD Healthcare, Wampsville, New York) combine high-frequency oscillation and positive expiratory pressure by employing a counterweighted lever and magnet. ${ }^{3}$ Exhaled air passes through a cone, which is intermittently occluded by a plug attached to the lever, producing air-flow oscillations. All 3 devices have 5 different settings, which can be selected by turning a wheel at the distal end of the device. This changes the proximity of the magnet and counterweighted plug, thereby adjusting the vibration frequency and amplitude, as well as the required mean pressure. ${ }^{3}$ The 3 different models are described as follows on the manufacturer's homepage: Acapella Choice, autoclave-able, for all patients; Acapella blue, for expiratory flows of $<15 \mathrm{~L} / \mathrm{min}$ (for $3 \mathrm{~s}$ ); and Acapella green, for expiratory flows of $>15 \mathrm{~L} / \mathrm{min}$ (for $3 \mathrm{~s}$ ).

\section{Water Bottle}

The water bottle used in this study was a 1.5 -L plastic bottle (conventional beverage bottle) with a medical-grade polyvinyl chloride tube of $50-60 \mathrm{~cm}$ in length and an inner diameter of $8 \mathrm{~mm}$. The bottle can be filled with different water volumes depending on the expiratory muscle strength of a patient. The required expiratory pressure increases with increasing water volumes.

\section{QUICK LOOK}

\section{Current knowledge}

A number of expiratory pressure and vibratory techniques have been developed to aid airway clearance in patients with retained secretions. The appropriate pressures and vibration frequencies have been explored but not clearly defined.

\section{What this paper contributes to our knowledge}

Compared to an expiratory water bottle system set at $5 \mathrm{~cm} \mathrm{H}_{2} \mathrm{O}$ pressure, the expiratory vibratory devices create a greater pressure amplitude within a frequency of $12-15 \mathrm{~Hz}$. This amplitude may enhance secretion removal.

\section{Measurement Setup and Data Acquisition}

All devices were connected to a gas flow and pressure analyzer (VT plus, BIO-TEK, Fluke Biomedical, Everett, Washington) and an adjustable constant air flow (Fig. 1). All 3 Acapella devices were tested in a horizontal position at flows of $6,12,20,30,40$, and $50 \mathrm{~L} / \mathrm{min}$, and at all 5 settings. The flows for testing the Acapella devices were chosen according to values used in clinical practice or published in the literature. ${ }^{4}$ Even though the Acapella devices are specified for different flows (green model, $>15 \mathrm{~L} /$ min; blue model, $<15 \mathrm{~L} / \mathrm{min}$ ), we tested all devices at all flows to have a direct comparison among the devices. The water bottle (1.5-L plastic bottle) was filled to water heights of 5,10 , or $15 \mathrm{~cm}$, and was tested at flows of $3,6,10,12$, and $20 \mathrm{~L} / \mathrm{min}$ because this device is usually used for patients with highly impaired expiratory function.

First, we determined the resistance of every measurement setup (ie, the gas flow analyzer and the tube with the connected device). The resistance of the measurement setup was subtracted from the measured resistance of each tested setting. The sampling frequency was $50 \mathrm{~Hz}$, and for each test setting $30 \mathrm{~s}$ of data were recorded and stored locally. We measured the frequency (in hertz) and amplitude (in centimeters of $\mathrm{H}_{2} \mathrm{O}$ ) of the oscillating air as well as the resulting pressure (in centimeters of $\mathrm{H}_{2} \mathrm{O}$ ) to generate the oscillation for all tested settings.

\section{Analysis}

The data stored locally in the gas flow analyzer were exported to a personal computer and stored as ASCII files. Further data processing of the ASCII files was performed with MatLab7 (MathWorks, Natick, Massachusetts). To have stable values, the data recorded between the 15th and the 20th second were used for further analysis. The means 


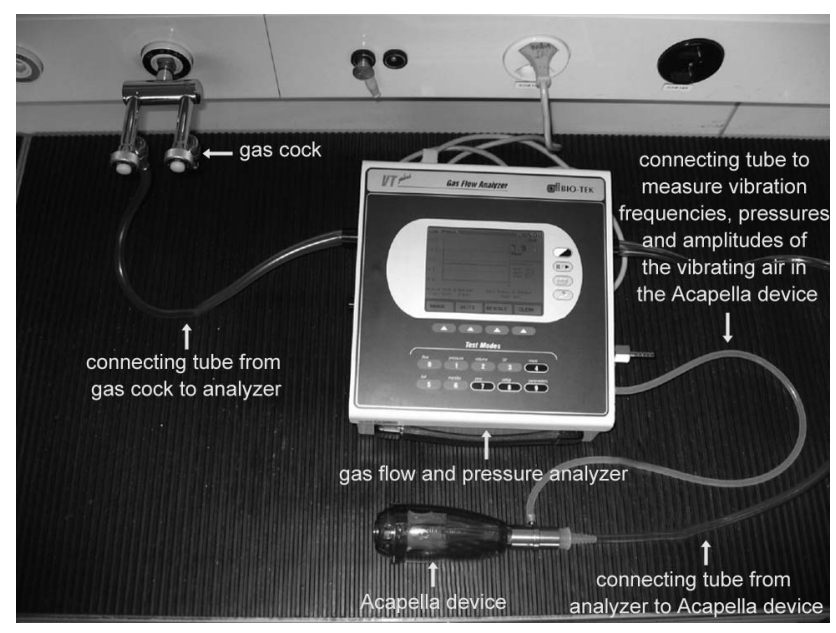

Fig. 1. Measurement setup: gas flow analyzer connected to an Acapella device.

of the data recorded between the 15th and 20th second were calculated and used for creating the figures. The frequencies of the oscillating air were calculated with a Fourier transformation using MatLab7. Flow, pressure, and amplitude data for each device and for all tested settings were also exported from the gas flow analyzer to MatLab. We generated figures of amplitudes, frequencies, and pressures versus flow for each device at all tested settings using Systat 10.2 (Systat Software, Chicago, Illinois). For determining the optimal settings (Acapella) or filling height (water bottle), we first selected oscillating air frequencies between 11 and $15 \mathrm{~Hz}(13 \pm 2 \mathrm{~Hz})$. Second, settings and flows corresponding to these frequencies were identified. Finally, the corresponding amplitudes of these settings and flows were analyzed. The optimal setting or filling height was determined by the flow generating the vibration frequency with the greatest amplitude within the target frequency range. Furthermore, the required pressure for generating this flow was identified.

\section{Results}

\section{Acapella}

The required expiratory pressure to generate vibrations increased more with higher flows than with increasing settings of the Acapella devices (Table 1).

The frequency-flow relationship of the Acapella blue device (Fig. 2, left) showed an inverse pattern compared to the Acapella Choice (see Fig. 2, middle) and the Acapella green device (see Fig. 2, right). Even though the amplitudes of the vibrating air did not show a clear pattern, the Acapella blue (Fig. 3, left) produced lower amplitudes compared to the Acapella Choice (see Fig. 3, middle) and the Acapella green (see Fig. 3, right).
Table 1. Required Pressures (Range of Means Over the Measured Time Period of the Five Different Settings) of the Three Acapella Devices to Generate Different Flows

\begin{tabular}{cccc}
\hline \hline \multirow{2}{*}{$\begin{array}{c}3 \\
\text { Llmin }\end{array}$} & \multicolumn{3}{c}{ Pressure, cm $\mathrm{H}_{2} \mathrm{O}$} \\
\cline { 2 - 4 } & Acapella Choice & Acapella Green & Acapella Blue \\
\hline 6 & $2-7$ & $2-7$ & $1-8$ \\
12 & $2-7$ & $4-7$ & $8-10$ \\
20 & $5-8$ & $5-8$ & $10-15$ \\
30 & $10-12$ & $7-12$ & $22-30$ \\
40 & $16-20$ & $15-20$ & $42-58$ \\
50 & $27-30$ & $21-27$ & $100-120$ \\
\hline
\end{tabular}

\section{Water Bottle}

Increasing the filling height of the water bottle increased the required pressure to generate a vibrating effect (filling height $=$ required pressure in centimeters of $\mathrm{H}_{2} \mathrm{O}$ ), under the condition that the end of the tube was at the bottom of the bottle. Clear vibration signals resulted only if the end of the rubber tube was in the proximity of the bottle wall and not if it was in the middle of the bottle. We recorded vibration frequencies in the target range $(13 \pm 2 \mathrm{~Hz})$ with a filling height of $5 \mathrm{~cm}$ and at a flow of $>6 \mathrm{~L} / \mathrm{min}$ (Fig. 4 , left). Unfortunately, all vibration amplitudes were much lower compared to the Acapella devices (see Fig. 4, right).

The optimal settings for the 4 tested devices are presented in Table 2.

\section{Discussion}

This study shows that the Acapella green device generates the greatest amplitudes in the optimal frequency range. To our knowledge there are only 2 other studies ${ }^{3,4}$ in which the Acapella devices have been evaluated concerning the optimal settings for generating vibrations effective for secretion mobilization. Volsko et $\mathrm{al}^{3}$ have only tested the Acapella blue device at low flows of 5-15 L/min and the Acapella green device at high flows of $20-30 \mathrm{~L} / \mathrm{min}$. Furthermore, they have only evaluated settings 1,3 , and 5 . Nevertheless, the mean pressures, amplitudes, and frequencies, which they have reported, are comparable to our results. In another study, ${ }^{4}$ the Acapella green device has been evaluated at all 5 settings for flows $(12-48 \mathrm{~L} / \mathrm{min})$ comparable to those in the present study. However, only the physical performance has been investigated. ${ }^{4}$ The reported frequencies, pressures, and amplitudes were in a similar range as our results, but the differences in pressure and amplitude among the 5 settings were smaller. ${ }^{4}$ Alves Silva et $\mathrm{al}^{4}$ have used 3 different Acapella green devices and calculated the means of the measured values, which may have smoothened the curves and resulted in discrepancies with the present results. Two further studies have 

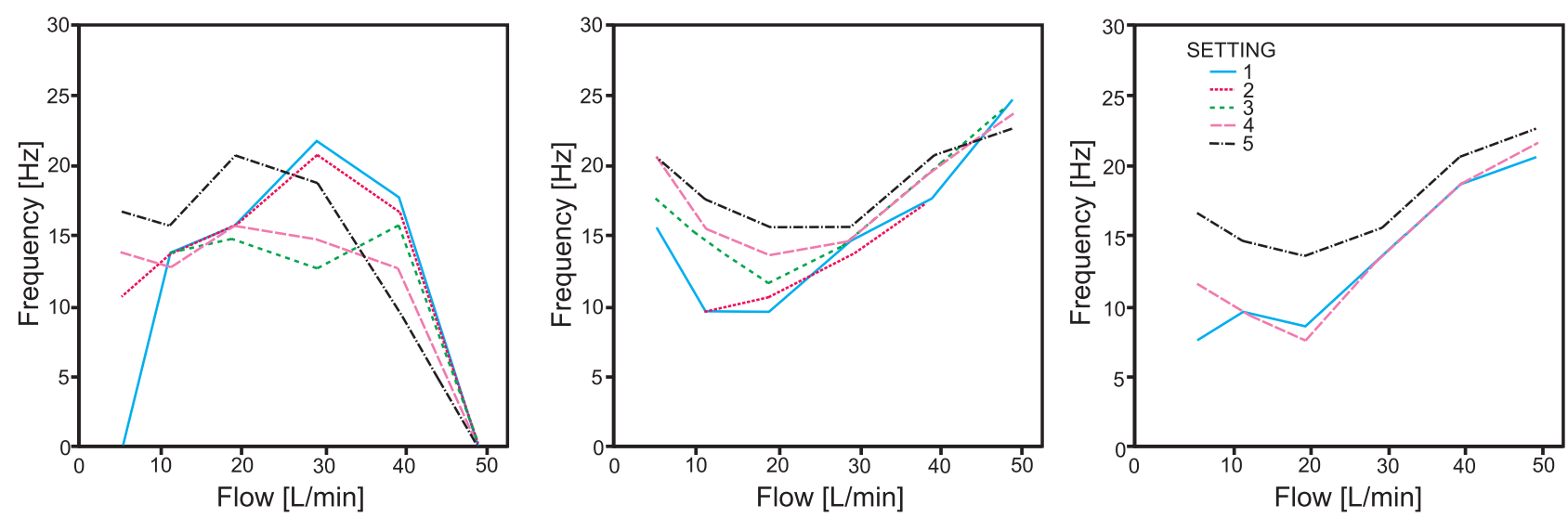

Fig. 2. Frequency-flow relationship at the five different settings and for all three Acapella devices (left: Acapella blue; middle: Acapella Choice; and right: Acapella Green). In the left and middle panels, parts of settings 2-4 overlay with other settings, and in the right panel, settings 2 and 3 completely overlay setting 1. Reported values are means over the analyzed time period at each setting and flow.
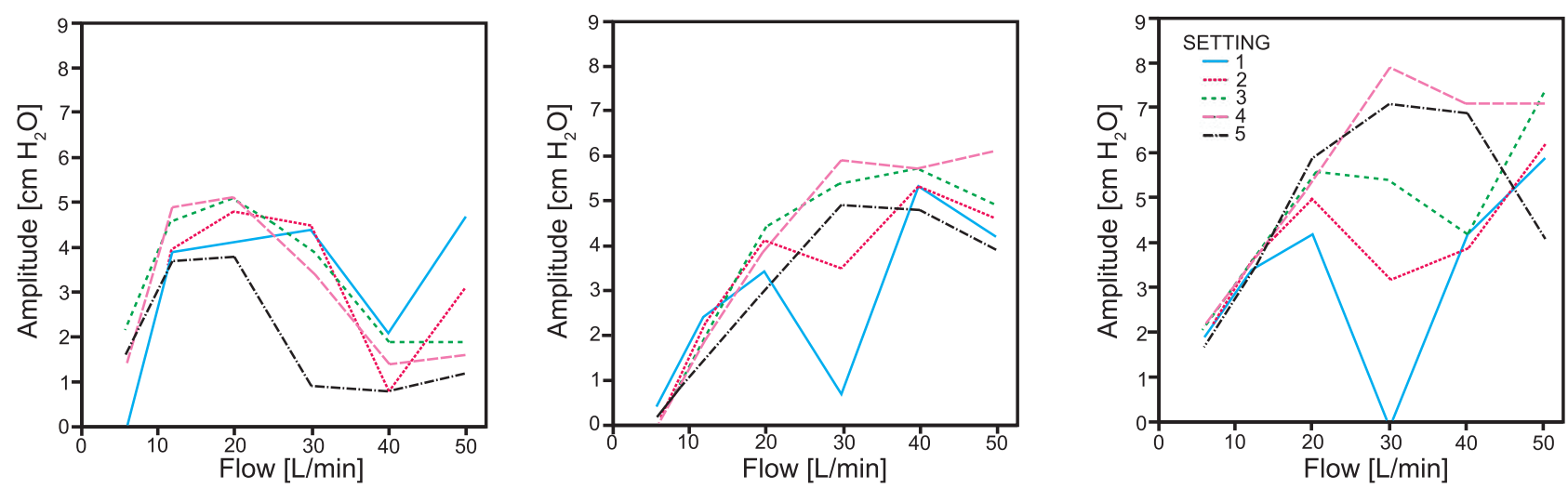

Fig. 3. Amplitude-flow relationship at the five different settings and for all three Acapella devices (left: Acapella blue; middle: Acapella Choice; and right: Acapella Green). Note that reported values are means over the analyzed time period at each setting and flow.
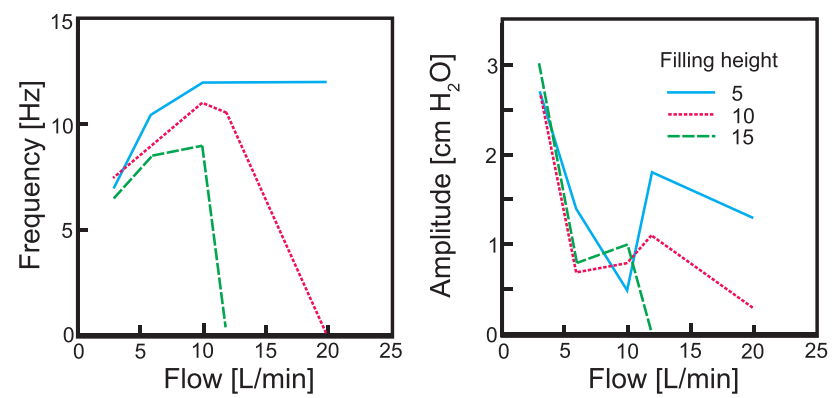

Fig. 4. Frequency-flow relationship (left) and amplitude-flow relationship (right) of the water bottle at all 3 filling heights $(\mathrm{cm})$. Note that reported values are means over the analyzed time period at each filling height and flow.

compared the Acapella device (model not specified) with normal airway clearance in clinical crossover trials. ${ }^{1,5}$ These articles have a high clinical relevance because they have assessed the expectorated sputum volumes. However, the Acapella device settings have not been reported, and it is therefore difficult to compare these results with ours. We
Table 2. Optimal Settings for the Acapella Devices and Optimal Water Filling Height for the Water Bottle (Pressures, Frequencies, and Amplitudes Are Means Over the Measured Time Period)

\begin{tabular}{cccccc}
\hline \hline Device & $\begin{array}{c}\text { Setting/Filling } \\
\text { Height, cm }\end{array}$ & $\begin{array}{c}\text { Flow, } \\
\text { L/min }\end{array}$ & $\begin{array}{c}\text { Pressure, } \\
\mathrm{cm} \mathrm{H}_{2} \mathrm{O}\end{array}$ & $\begin{array}{c}\text { Frequency, } \\
\mathrm{Hz}\end{array}$ & $\begin{array}{c}\text { Amplitude, } \\
\mathrm{cm} \mathrm{H}_{2} \mathrm{O}\end{array}$ \\
\hline $\begin{array}{c}\text { Acapella } \\
\text { Choice }\end{array}$ & 4 & 30 & 11 & 15 & 8 \\
$\begin{array}{c}\text { Acapella } \\
\text { blue }\end{array}$ & 4 & 12 & 8 & 13 & 5 \\
$\begin{array}{c}\text { Acapella } \\
\text { green }\end{array}$ & 4 & 30 & 11 & 13 & 6 \\
$\begin{array}{c}\text { Water } \\
\text { bottle }\end{array}$ & 5 & 12 & 5 & 12 & 1.8 \\
\hline
\end{tabular}

are the first to evaluate the Acapella Choice device, which has the advantage that it can be sterilized. However, in the light of our results, the usefulness of the Acapella Choice is questionable because it produces vibrations with higher frequencies and smaller amplitudes, which are less optimal 
for secretion mobilization, than the Acapella green device (Table 2), and it has the same pressure and flow requirements as the Acapella green device.

Another important factor for determining optimal settings for secretion mobilization devices is the natural frequency of the chest, pulmonary tissue, and respiratory tract. ${ }^{8}$ Cegla and Retzow ${ }^{9}$ have reported that natural lung-chest frequencies vary between 12 and $15 \mathrm{~Hz}$. According to de Lima et $\mathrm{al}^{8}$ the natural frequency also depends on the postural position (ie, sitting or standing) and is lower in patients with respiratory illness like, for example, cystic fibrosis. The literature on this topic is very sparse and mainly supports frequencies in the target range chosen in our study (ie, $11-15 \mathrm{~Hz}$ ). ${ }^{1,8,9}$

We have also evaluated the vibration properties of the water bottle, because this is a tool often used in clinical practice and by patients at home, and it is cheap and easily obtainable. However, an increased infection risk may develop if the water is not changed daily, and if the bottle as well as the tube are not exchanged regularly. To our knowledge no literature exists concerning the optimal settings of the water bottle. Like the Acapella devices, the water bottle can be used at different settings (eg, filling height, flows, placement of the tube), and the evaluation of the optimal settings is therefore justified. Our results clearly demonstrate that the water bottle may not be effective in all patients, because it generates only very small amplitudes at which optimal secretion mobilization is questionable. However, the water bottle has the advantage that it can be used with a water height of only $5 \mathrm{~cm}$, which requires a very low expiratory muscle strength and is therefore suitable for patients who are not yet able to use the Acapella blue device. The water bottle may also be an effective tool developing countries if basic hygienic principles are followed and access to clean water is ensured.

We suggest the following approach for the clinical application of these devices. Acapella green seems to be the best device when patients are able to sustain a minimal expiratory pressure of $11 \mathrm{~cm} \mathrm{H}_{2} \mathrm{O}$ with a flow of $30 \mathrm{~L} / \mathrm{min}$ for at least $3 \mathrm{~s}$. The Acapella blue device may be optimal for weaker patients who are able to generate expiratory pressures of $8-10 \mathrm{~cm} \mathrm{H}_{2} \mathrm{O}$ at flows of only $12 \mathrm{~L} / \mathrm{min}$. Interestingly, all Acapella devices show their best vibration characteristics at setting 4 . Even though the vibration amplitude of the water bottle is rather small, it may be used in very weak patients with expiratory pressures of only $5-7 \mathrm{~cm} \mathrm{H}_{2} \mathrm{O}$ to start therapy for secretion mobilization as early as possible.

\section{Limitations}

The limitations of this study are that the present measurements have been made in a laboratory setting, and the results are therefore theoretical optimal settings, which have to be confirmed in patients in a clinical setting. However, this was the first step in the evaluation of the optimal device settings. Furthermore, the optimal frequency and intensity of the therapy are still unknown, and the response of individual patients may vary at different flows and amplitudes depending on the degree of air-flow obstruction and/or disease specification and severity. Concerning the water bottle, we did not investigate and determine the optimal size of the bottle, and the length and placement of the tube. Nevertheless, the required pressure is only determined by the water-filling height, and thus the size of the bottle does not seem to play an important role.

\section{Conclusions}

The Acapella devices seem to be more efficient for secretion mobilization than the water bottle, because they generate greater vibration amplitudes. We suggest the use of the Acapella green device as soon as patients are able to sustain a minimal pressure of $11 \mathrm{~cm} \mathrm{H}_{2} \mathrm{O}$ with a flow of $30 \mathrm{~L} / \mathrm{min}$ for at least $3 \mathrm{~s}$. Generally, all 3 Acapella devices should be used at setting 4 , because frequencies and vibration amplitudes are optimal only at this setting.

\section{ACKNOWLEDGMENT}

We thank Jörg Krebs, Clinical Trial Unit, Swiss Paraplegic Centre, Nottwil, Switzerland, for critical reading of this manuscript.

\section{REFERENCES}

1. Patterson JE, Hewitt O, Kent L, Bradbury I, Elborn JS, Bradley JM. Acapella versus "usual airway clearance" during acute exacerbation in bronchiectasis: a randomized crossover trial. Chron Respir Dis 2007;4(2):67-74.

2. Alves LA, Pitta F, Brunetto AF. Performance analysis of the Flutter VRP1 under different flows and angles. Respir Care 2008;53(3):316323.

3. Volsko TA, DiFiore J, Chatburn RL. Performance comparison of two oscillating positive expiratory pressure devices: Acapella versus Flutter. Respir Care 2003;48(2):124-130.

4. Alves Silva CE, Santos JG, Jansen JM, de Melo PL. Laboratory evaluation of the Acapella device: pressure characteristics under different conditions, and a software tool to optimize its practical use. Respir Care 2009;54(11):1480-1487.

5. Patterson JE, Bradley JM, Hewitt O, Bradbury I, Elborn JS. Airway clearance in bronchiectasis: a randomized crossover trial of active cycle of breathing techniques versus Acapella. Respiration 2005; 72(3):239-242.

6. Volsko TA. The value of conducting laboratory investigations on airway clearance devices. Respir Care 2008;53(3):311-313.

7. Brooks D, Newbold E, Kozar LF, Rivera M. The Flutter device and expiratory pressures. J Cardiopulm Rehabil 2002;22(1):53-57.

8. de Lima LC, Duarte JB, Lepore Neto FP, Abe PT, Gastaldi AC. Mechanical evaluation of a respiratory device. Med Eng Phys 2005; 27(2):181-187.

9. Cegla UH, Retzow A. Physical therapy with VRP1 in chronic obstructive respiratory tract diseases-results of a multicenter comparative study. Pneumologie 1993;47(11):636-639. 